\title{
Xuan Bai Cheng Qi formula as an adjuvant treatment of acute exacerbation of chronic obstructive pulmonary disease of the syndrome type phlegm-heat obstructing the lungs: a multicenter, randomized, double-blind, placebo-controlled clinical trial
}

Miao Liu, Xianggen Zhong, Yuhang Li ${ }^{*}$, Fengjie Zheng, Ruohan Wu, Yan Sun and Jinchao Zhang

\begin{abstract}
Background: Acute exacerbation of chronic obstructive pulmonary disease (AECOPD) is a common cause of morbidity and mortality. Traditional Chinese medicine (TCM) is used to treat AECOPD as adjunctive therapy. This study aimed to evaluate the efficacy and safety of the TCM formula Xuan Bai Cheng Qi as an adjuvant therapy for AECOPD patients with the syndrome type of phlegm-heat obstructing the lungs.

Methods: A multicenter, randomized, double-blind, placebo-controlled clinical trial was conducted. A total of 244 patients were divided into the intervention group $(n=122$, treated with conventional medicine and Xuan Bai Cheng Qi) and the control group ( $n=122$, treated with conventional medicine and placebo). Total symptom scores (cough, phlegm, wheezing, chest congestion) before treatment and at 3, 5, 7, 10 days post-treatment were recorded. Lung function, arterial blood gas, serum inflammatory cytokines, oxidation/anti-oxidation index were observed before treatment and at the end of the 10-day treatment.

Results: A total of 242 patients completed the study. The full analysis set (FAS) population was 244 and the per-protocol analysis set (PPS) population was 229. After the 10-day treatment, symptom scores of the Xuan Bai Cheng Qi group were significantly lower over time compared with the control group (FAS: mean difference -1.84 , 95\% Cl -2.66 to $-1.03, P<.001$; PPS: mean difference $-1.87,95 \% \mathrm{Cl}-2.71$ to $-1.03, P<.001)$. FEV 1 , FVC, and FEV $1 \%$ pred were significantly higher over time in the Xuan Bai Cheng Qi group compared with those in the control group (day 10, FAS and PPS: $P<.05$ ). $\mathrm{PaO}_{2}$ and $\mathrm{PaCO}_{2}$ were significantly improved in the Xuan Bai Cheng Qi group (day 10, FAS and PPS: $P<.05$ ). Xuan Bai Cheng Qi was also found to ameliorate cytokine levels and oxidation/antioxidant index compared with placebo. There were no differences in safety variables and adverse events between the two groups.
\end{abstract}

Conclusions: Xuan Bai Cheng Qi formula appears to be a safe and beneficial treatment for AECOPD of phlegm-heat obstructing the lungs syndrome type.

Keywords: Acute exacerbation, Chronic obstructive pulmonary disease, Xuan Bai Cheng Qi, Phlegm-heat obstructing lung, Syndrome, Traditional Chinese medicine

\footnotetext{
* Correspondence: liyuhang@bucm.edu.cn

School of Preclinical Medicine, Beijing University of Chinese Medicine, 11 East Third Ring Road, Beijing 100029, China
} 


\section{Background}

Chronic obstructive pulmonary disease (COPD) is defined by irreversible or partially reversible airway obstruction in persons with chronic bronchitis or emphysema. Experts predict that COPD will become the fourth leading cause of death worldwide by 2030 [1].

Hastening of normal reduction in lung function that occurs with age and repeated exacerbations are key features of COPD. Acute exacerbation of chronic obstructive pulmonary disease (AECOPD) is a common cause of morbidity and mortality in COPD patients [2]. These exacerbations are associated with worsening respiratory symptoms and lung function [3]. Median time postexacerbation to recovery of peak flow is 6 days and time to recovery from symptoms is 7 days [4].

Many etiologic factors contribute to COPD exacerbations such as continued smoking, respiratory tract infections, and exposure to particles from air pollution and the work environment. Upper respiratory tract infections are the most common triggering factor of COPD exacerbations. Thus, the management of exacerbations creates an enormous burden on health care services [5].

Traditional Chinese medicine (TCM) is used extensively in the treatment of AECOPD in Asia, particularly in China. The effects of TCM, especially herbal medicines, in improving respiratory symptoms and decreasing the incidence of COPD exacerbations are well known. One such herbal medicine is Xuan Bai Cheng Qi formula. In Chinese medicine, the primary syndrome type of AECOPD is phlegm-heat obstructing the lungs [6]. The actions of Xuan Bai Cheng Qi formula are to clear lung heat and dissipate phlegm, so for this reason it is commonly prescribed for AECOPD [7]. The remedy consists of four Chinese herbs: Gypsum fibrosum (gypsum), Rheum officinale Baill (rhubarb root and rhizome), Armeniacae amarum (apricot seed or kernel), and Trichosanthes kirilowii (trichosanthes peel) (Table 1). Xuan Bai Cheng Qi is a classical prescription dating to the Qing Dynasty (late 1700s) text Systematic Differentiation of Warm Pathogen Diseases by Wu Jutong. Our previous studies found that Xuan Bai Cheng Qi improves oxidant/ antioxidant imbalance, pulmonary inflammation in the rat model of COPD $[8,9]$. Clinical studies have postulated that Xuan Bai Cheng Qi is effective as a supplemental remedy for treating AECOPD or respiratory failure [10,11]. However, due to the limited sample sizes and methodological problems apparent in these studies the evidence for the effectiveness of Xuan Bai Cheng Qi was not robust. Therefore, we performed a multicenter, randomized, double-blind, placebo-controlled clinical trial to evaluate the efficacy and safety of Xuan Bai Cheng Qi in the treatment of AECOPD of the syndrome type phlegmheat obstructing the lungs.

\section{Methods}

\section{Ethics and trial registration}

This study was approved by the research ethics committees at all participating centers and on file at Dongzhimen Hospital affiliated to Beijing University of Chinese Medicine (DZMSP20090302). This trial was registered with the Chinese Clinical Trial Registry (ChiCTR-TRC09000533).

\section{Patients}

\section{Diagnostic criteria}

AECOPD diagnosis was based on criteria established by the Global Initiative for Chronic Obstructive Lung Disease (GOLD, 2007 version) [12], and by the Chinese Society of Respiratory Diseases (2007 version) [13].

Diagnostic criteria for TCM syndrome of phlegm-heat obstructing lung was based on State Administration of Traditional Chinese Medicine guidelines [14] and criteria outline by Wang and Lu [15]. Primary symptoms were: cough and/or gasping for breath; yellow and viscous sputum. Accompanying symptoms were: irritability and restlessness; abdominal distension; thirst with desire for cool drink; fever without chills; yellow urine; constipation with hard stool; red tongue with yellow or yellow greasy coating; slippery and rapid pulse. Phlegm-heat obstructing the lungs was diagnosed when any two primary symptoms along with any two accompanying symptoms were present.

\section{Inclusion and exclusion criteria}

Persons of either gender were included in the study if they: met the diagnostic criteria of AECOPD; met TCM diagnostic criteria for syndrome of phlegm-heat obstructing lung; had symptoms defined by GOLD as level 1 to 4;

Table 1 Medicinals in Xuan Bai Cheng Qi formula

\begin{tabular}{|c|c|c|c|c|}
\hline Chinese name (pinyin) & Latin name & English common name & $\begin{array}{l}\text { Daily decoction } \\
\text { dose }^{a}(\mathrm{~g})\end{array}$ & $\begin{array}{l}\text { Dose per } 1,000 \mathrm{~g} \\
\text { granule }^{\mathrm{b}}(\mathrm{g})\end{array}$ \\
\hline Sheng Shi Gao & Gypsum fibrosum & Gypsum & 30 & 435 \\
\hline Sheng Dahuang & Rheum officinale Baill & Unprocessed rhubarb root and rhizome & 18 & 261 \\
\hline Ku Xing Ren & Armeniacae amarum & Bitter apricot seed & 12 & 174 \\
\hline Gua Lou Pi & Trichosanthes kirilowii & Trichosanthes peel & 9 & 130 \\
\hline
\end{tabular}

${ }^{a}$ Amount of each medicinal if formula were prepared as a decoction.

${ }^{\mathrm{b}}$ Amount of each medicinal used in preparing granule form of this formula. 
were aged between 18 to 85 years; were within 5 days post-AECOPD; had not participated in other interventional trials in the previous 1 month; and were willing to participate in the study voluntarily and signed an informed consent.

Persons were excluded from the study if they had any of the following: airflow limitation due to bronchiectasis, cystic fibrosis, lung cancer, or other respiratory disease; co-morbidities such as acute heart failure, acute cerebral hemorrhage, gastrointestinal bleeding, aplastic anemia, or other severe or life-threatening diseases; unstable hypertension, coronary heart disease, or diabetes; mental disease, severe neurologic deficits or other disorders that impaired understanding or cooperation with the investigation; serious liver, kidney, or hematopoietic disease; recent use of immunosuppressive agents; known allergy to herbs or drugs used in the trial. Women planning to become or were pregnant or breastfeeding were also excluded.

\section{Participant enrollment}

Participants in the trial were inpatients diagnosed with AECOPD of the syndrome phlegm-heat obstructing the lungs. They were recruited and enrolled on a rolling basis and observed from October 2009 through January 2013. Eight teaching hospitals in China were a part of the trial: Dongzhimen Hospital affiliated to Beijing University of Chinese Medicine (40 cases; 16.4\%); Dongfang Hospital affiliated to Beijing University of Chinese Medicine (26 cases; 10.7\%); Shanghai Shuguang Hospital affiliated to Shanghai University of Chinese Medicine (45 cases; 18.4\%); University Hospital of Gansu Traditional Chinese Medicine (10 cases; 4.1\%); First Affiliated Hospital of Anhui University of Chinese Medicine (23 cases; 9.4\%); Hebei Provincial Hospital of Chinese Medicine (38 cases; 15.6\%); Henan Province Kaifeng City Hospital of Traditional Chinese Medicine (18 cases; 7.4\%);
Affiliated Hospital of Liaoning University of Traditional Chinese Medicine (44 cases; 18.0\%).

\section{Treatments \\ Conventional therapy}

All participants in the trial and control groups were given conventional treatment based on GOLD [12] and the Chinese Society of Respiratory Diseases [13]. Conventional medicine regimen: oxygen therapy $(1-3 \mathrm{~L} / \mathrm{min})$ by nasal cannula or Venturi mask; open-label cefotaxime 2 g daily (iv q $12 \mathrm{~h}$ ) for 10 days or an alternate antibiotic if the patient was allergic to or intolerant of cefotaxime (Table 2); inhaled long-acting $\beta$-agonist (either salmeterol or formoterol) and an inhaled long-acting anticholinergic bronchodilator (tiotropium) after randomization; if the patient was using an inhaled steroid or inhaled steroid/ long-acting $\beta$-agonist combination product at the time of randomization, this medication was continued for patients in both groups; if the patient's baseline $\mathrm{FEV}_{1}$ was $<50 \%$ of predicted, 30-40 mg prednisolone per day for 7-10 days was administered. Supplemental salbutamol, ambroxol, and aminophylline were given as needed throughout the trial period.

\section{Experimental and control treatments}

Participants in the intervention group received Xuan Bai Cheng Qi formula in granule form as an adjunct to conventional therapy (Table 1). Participants in the control group received conventional therapy plus placebo granules.

\section{Manufacturing and administration of Xuan Bai Cheng Qi and placebo granules}

Both Xuan Bai Cheng Qi granules and the placebo granules were produced and packaged by Jiang Yin Tian Jiang Pharmaceutical Co. Ltd. (Jiangsu, China) under

Table 2 Application of antibiotics at each stage of AECOPD

\begin{tabular}{|c|c|c|}
\hline $\begin{array}{l}\text { COPD stage of acute } \\
\text { exacerbation }\end{array}$ & Microorganisms & Antibiotics \\
\hline GOLD stages I and II & $\begin{array}{l}\text { Haemophilus influenzae, Streptococcus pneumoniae, } \\
\text { Moraxella catarrhalis }\end{array}$ & $\begin{array}{l}\text { Penicillin, beta lactamase/enzyme inhibitor (amoxicillin/ } \\
\text { clavulanic acid), macrolides (clarithromycin, roxithromycin, } \\
\text { azithromycin), first generation (cefazolin, cefradine) or } \\
\text { second generation cephalosporins (cefuroxime, cefaclor), } \\
\text { doxycycline, levofloxacin. }\end{array}$ \\
\hline $\begin{array}{l}\text { GOLD stages III and IV; no risk } \\
\text { factors for infection with } \\
\text { Pseudomonas aeruginosa }\end{array}$ & $\begin{array}{l}\text { Haemophilus influenzae, Streptococcus pneumoniae, } \\
\text { Moraxella catarrhalis, Klebsiella pneumoniae, } \\
\text { Escherichia coli, Enterobacteriacea }\end{array}$ & $\begin{array}{l}\text { Beta-lactam/enzyme inhibitor (cefuroxime), second } \\
\text { generation cephalosporins, fluoroquinolones (levofloxacin, } \\
\text { moxifloxacin, gatifloxacin), third generation cephalosporins } \\
\text { (ceftriaxone, cefotaxime). }\end{array}$ \\
\hline $\begin{array}{l}\text { GOLD stages III and IV; risk } \\
\text { factors for infection with } \\
\text { Pseudomonas aeruginosa }\end{array}$ & $\begin{array}{l}\text { All above microorganisms plus Pseudomonas } \\
\text { aeruginosa }\end{array}$ & $\begin{array}{l}\text { Third generation cephalosporins (ceftazidime), } \\
\text { cefoperazone/sulbactam, piperacillin/tazobactam, imipenem, } \\
\text { meropenem, can also be combined with aminoglycosides, } \\
\text { fluoroquinolones (ciprofloxacin). }\end{array}$ \\
\hline
\end{tabular}


good manufacturing practice regulations of China (Approval Number: 0905301-4).

Xuan Bai Cheng Qi granules were prepared by decocting the four herbs together, resulting in a concentrated liquid and extraction and capture of the volatile oils. The concentrated liquid was spray-dried on a starch base powder to obtain the final product. Each package of granules was 4.5 g. Quality of the granules was tested for consistency with industry standards.

The placebo granules were designed to taste, smell, and resemble the Xuan Bai Cheng Qi granules.

Participants were given packages of Xuan Bai Cheng Qi or placebo granules to prepare on their own over the 10-day trial period. Four packages were to be taken each day by dissolving the granules in $300 \mathrm{~mL}$ boiled water with $150 \mathrm{~mL}$ taken twice daily. To assess adherence, at the completion of the trial on day 10, participants turned in used and unused packages of granules to research team nurses.

At the start of the trial, participants were instructed not to receive other TCM therapy associated with the treatment of AECOPD.

\section{Outcome measures}

A case report form (CRF) was designed by the research steering committee. Spot checks were conducted throughout the trial by seven independent reviewers from within the steering committee to check completion of the CRF and conformance to the protocol. Randomly selected CRFs were reviewed during the trial to ensure quality.

Primary outcome measure was symptom scores, which were assessed and recorded on day 1, 3, 5, 7, 10. Secondary outcome measures were lung function, arterial blood gas analysis, pro-inflammatory biomarkers (cytokines) and oxidation/antioxidant index, which were measured and recorded on days 1 and 10 .

\section{Symptom scores}

A symptom score sheet was designed based on the typical format of a 4-point Likert scale [16] with questions pertaining to four AECOPD symptoms in terms of TCM syndrome of phlegm-heat obstructing the lung (cough, phlegm, wheezing, chest congestion) [17]. Members of the research team administered the questionnaire to participants. Severity of symptoms was assigned the following points: 0 (none), 2 (mild), 4 (moderate), and 6 (severe). Total score range was from 0 (asymptomatic) to 30 (severe).

\section{Lung function}

Lung function was evaluated in participants using spirometry. Forced expiratory volume in one second $\left(\mathrm{FEV}_{1}\right)$, forced vital capacity (FVC) and $\mathrm{FEV}_{1}$ percentage of the predicted value $\left(\mathrm{FEV}_{1} \%\right.$ pred) were tested.

\section{Arterial blood gas analysis}

Arterial blood gas parameters that were measured were $\mathrm{pH}$, arterial partial pressure of oxygen $\left(\mathrm{PaO}_{2}\right)$, arterial partial carbon dioxide pressure $\left(\mathrm{PaCO}_{2}\right)$.

\section{Pro-inflammatory biomarkers (cytokines)}

Levels of tumor necrosis factor alpha (TNF- $\alpha$ ), interleukin4 (IL-4), interleukin-8 (IL-8), interleukin-1 beta (IL-1 $\beta$ ), interleukin-6 (IL-6), interleukin-2 (IL-2) in serum were quantified using an enzyme-linked immunosorbent assay (ELISA) kit (BD Biosciences, San Diego, CA).

\section{Oxidant/antioxidant balance}

Levels of superoxide dismutase (SOD) and malondialdehyde (MDA) in serum were quantified using commercially available kits (Nanjing Jiancheng BioEngineering Institute, Nanjing, China).

\section{Safety variables}

Complete blood count, urine, liver and kidney function tests, and electrocardiography were performed pre- and post-treatment (days 1 and 10). Any adverse events that occurred during the treatment period were recorded.

\section{Adherence}

Treatment adherence was assessed by investigators at each center counting used and unused granule packages.

\section{Study design}

This was a multicenter, randomized, double-blind, placebocontrolled, parallel group clinical trial.

\section{Sample size}

As an exploratory study, reference power calculations were lacking as there have been only a few small-size trials in China of Xuan Bai Cheng Qi formula for treatment of AECOPD. Therefore, we inferred data from literature on TCM therapies with similar efficacy as Xuan Bai Cheng Qi that treated the same syndrome of phlegm-heat obstructing the lungs.

The approximate calculation of sample size was determined based on the effective rate of symptom scores (= (total scores of baseline - total scores of end)/total scores of baseline $\times 100 \%$ ) [18]. The effective rate of conventional medicine plus placebo $(75 \%)$ and the effective rate of conventional medicine plus Xuan Bai Cheng Qi (90\%) were based on comparable studies [19,20]. The control group and trial group adopted 1:1 parallel design. With 5\% one-sided significance level and $90 \%$ statistical power, it was determined that 122 participants should be randomly assigned to each group, considering an approximate $10 \%$ drop-out rate. 


\section{Randomization and blinding}

Participants were randomly allocated to receive Xuan Bai Cheng Qi formula or placebo. Randomization was achieved using SAS 8.0 software to generate random numbers. Simple randomization was used, with each block comprised of eight participants. Randomization numbers were assigned to participants in chronological order after their screening assessments. Participants in the active arm received Xuan Bai Cheng Qi formula (granules) plus conventional therapy. Participants in the inactive arm received conventional therapy plus matching placebo.

To ensure allocation concealment, opaque and sealed emergency letters were printed according to randomized list. In the event of an emergency, the participant's randomization code and group allocation could be identified by the emergency envelope, which were on file at all centers. Third-party investigators were assigned in each research center as the contact person who preserved and recorded the randomization information. They did not have contact with other trial investigators, therefore, they did not affect enrollment or randomization.

Xuan Bai Cheng Qi and placebo granules were repackaged in identical wrappers at each center before distribution to participants, and numbered sequentially according to randomization schedule. Outcome assessments were made by an independent statistician who was blinded to group allocation, intervention, and trial management and did not take part in any other part of the trial. Both

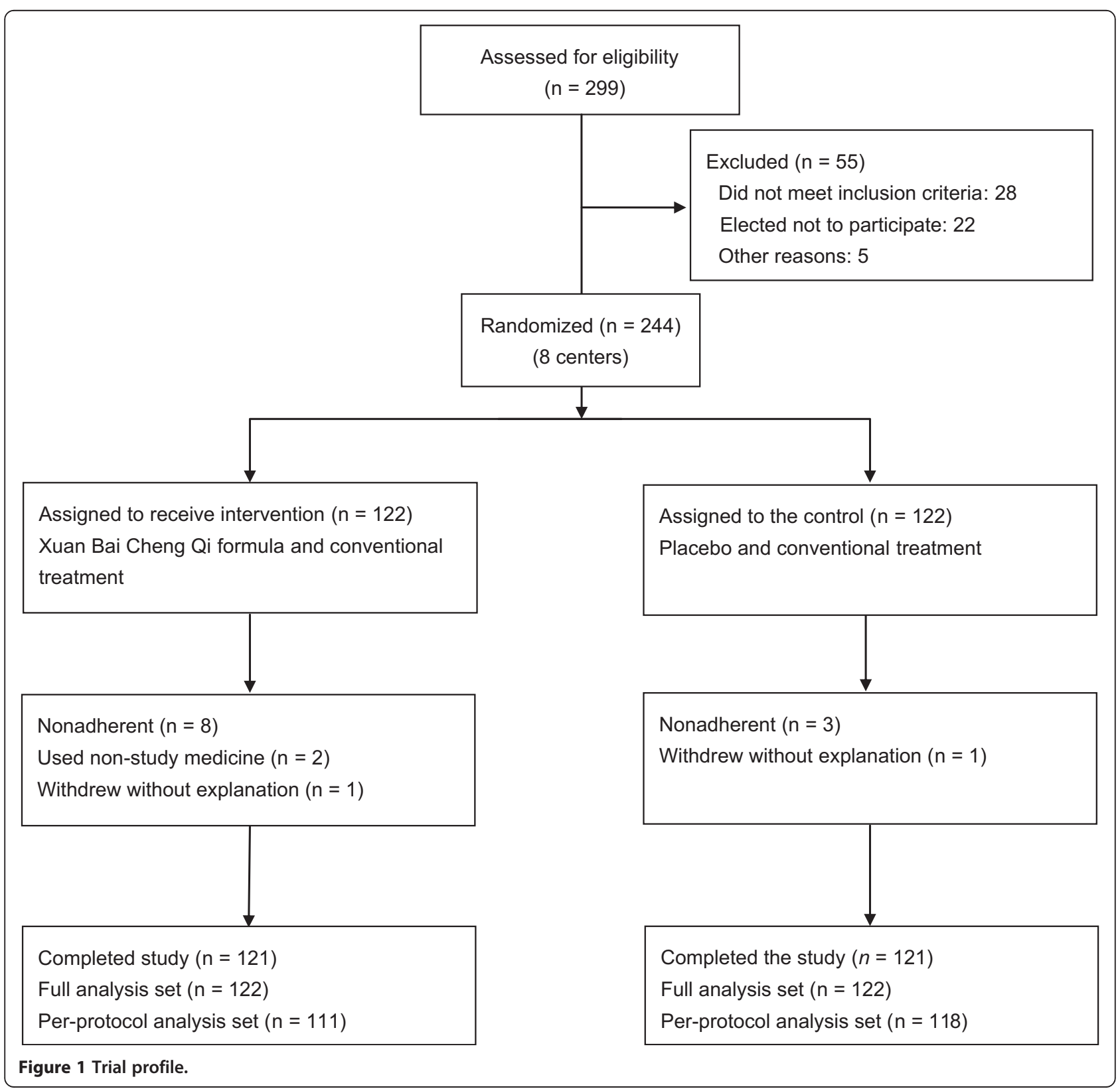


participants and investigators were blinded until the end of study.

\section{Statistical analysis}

All data were entered with Epidata 3.0 software (The EpiData Association, Odense, Denmark) by two independent data recorders. All randomized patients were included in data analysis. Full analysis set (FAS) was used to analyze the baseline data and outcome data of the cases that went through randomization and received treatment. Each participant underwent at least one time-point observation during the trial. Partially missing data were imputed with the use of the last-observation-carried-forward (LOCF) method, whereby missing values were replaced by the last non-missing value. Per-protocol analysis set (PPS) was used to analyze the evaluation data of cases that completed the trial without major protocol deviations.

Continuous variables were analyzed using repeatedmeasures analysis of variance (ANOVA) and/or analysis of covariance. Mauchly's test was used to assess sphericity in the repeated measures ANOVAs, and the Greenhouse Geisser correction was applied to the degrees of freedom (DFs) if necessary. Main effects for time, for the interaction between time and the intervention group, and for the overall difference between groups (Xuan Bai Cheng Qi versus placebo) were assessed. Categorical variables were analyzed using chi-square test, Fisher's exact test, or Cochran-Mantel-Haenszel test. The effect size estimate and 95\% confidence interval (CI) were applied for comparing the treatments. All statistical procedures were performed with SPSS 17.0 software (SPSS Inc, Chicago, IL).

\section{Data monitoring committee}

A data monitoring committee at Dongzhimen Hospital comprised of clinicians, statisticians, epidemiologists oversaw data analyses and ensured protocol adherence and safety of the trial. Data were reviewed and monitored every 6 months.

\section{Results}

\section{Study population}

A total of 299 COPD patients were assessed for eligibility in the trial. After 55 patients were excluded due to not meeting eligibility criteria, 244 patients were enrolled and underwent randomization (Figure 1). Two participants dropped out on day 7 without explanation, but they received treatment and also completed one time point observation. Thus, a total of 242 evaluable participants were in the study, with 121 in the Xuan Bai Cheng Qi group and 121 in the control group. The FAS population was 244, with 122 in the trial group and 122 in the control group. Eleven participants were protocol noncompliant, and two participants used non-study medications, which affected outcome calculation. Ultimately,
PPS population was 229, with 111 in the trial group and 118 in the control group.

In terms of demographic and baseline characteristics of participants (Table 3), there were no significant differences between the Xuan Bai Cheng Qi and control groups in gender, age, course of disease, body mass index (BMI), exacerbations, $\mathrm{FEV}_{1} / \mathrm{FVC}$, and GOLD classification of lung function.

\section{Symptom scores}

ANOVA revealed a significant main effect of group $(P=.001)$, a significant main effect of time $(P<.001)$, as well as a significant group $\times$ time interaction $(P<.001)$. Total symptom scores of the Xuan Bai Cheng Qi group were significantly lower over time compared with those of the control group (FAS: mean difference -1.84, 95\% CI -2.66 to $-1.03, P<.001$; PPS: mean difference -1.87 , $95 \% \mathrm{CI}-2.71$ to $-1.03, P<.001)$. This difference was achieved within the first 3 days of treatment (FAS: mean difference $-2.57,95 \% \mathrm{CI}-3.64$ to $-1.49, P<.001$; PPS: mean difference $-2.60,95 \% \mathrm{CI}-3.70$ to $-1.49, P<.001)$. At days 3, 5, 7, and 10, there were significant differences in total symptom scores of the Xuan Bai Cheng Qi group compared with those of the control group (Table 4).

\section{Table 3 Baseline characteristics of participants}

\begin{tabular}{|c|c|c|}
\hline & $X B C Q(n=122)$ & Control $(n=122)$ \\
\hline Age (SD), y & $70.1 \pm 9.8$ & $70.7 \pm 9.8$ \\
\hline Body mass index (SD), kg/m² & $23.36 \pm 3.44$ & $22.76 \pm 3.11$ \\
\hline \multicolumn{3}{|l|}{ Gender (male/female) } \\
\hline Male (n) \% & $88(72.1 \%)$ & $82(67.2 \%)$ \\
\hline Female (n) \% & $34(27.9 \%)$ & $40(32.8 \%)$ \\
\hline Body temperature $\left({ }^{\circ} \mathrm{C}\right)$ & $36.68 \pm 0.56$ & $36.66 \pm 0.58$ \\
\hline \multicolumn{3}{|l|}{ Exacerbation } \\
\hline Frequency (times) & $2.8 \pm 1.28$ & $2.6 \pm 1.24$ \\
\hline Currently exacerbation & $3.5 \pm 1.36$ & $3.5 \pm 1.29$ \\
\hline \multicolumn{3}{|l|}{ Smoking status } \\
\hline Currently smoking, n (\%) & $22(18.03 \%)$ & $16(13.11 \%)$ \\
\hline Never smoked, n (\%) & $54(44.26 \%)$ & $52(42.62 \%)$ \\
\hline Ever smoked, n (\%) & $46(37.70 \%)$ & $54(44.26 \%)$ \\
\hline No. of packs/year & $299.8 \pm 170.0$ & $337.9 \pm 192.6$ \\
\hline $\mathrm{FEV}_{1} / \mathrm{FVC}$ & $55.02 \pm 10.477$ & $56.91 \pm 9.592$ \\
\hline \multicolumn{3}{|l|}{ GOLD classification } \\
\hline GOLD stage I, n (\%) & $10(8.20 \%)$ & $9(7.38 \%)$ \\
\hline GOLD stage II, n (\%) & $56(45.90 \%)$ & $58(47.54 \%)$ \\
\hline GOLD stage III, n (\%) & $47(38.52 \%)$ & $45(36.89 \%)$ \\
\hline GOLD stage IV, n (\%) & $9(7.38 \%)$ & $10(8.20 \%)$ \\
\hline
\end{tabular}

Abbreviations: COPD chronic obstructive pulmonary disease, $F E V_{1}$ forced expiratory volume in one second, FVC forced vital capacity, GOLD Global Initiative for Chronic Obstructive Lung Disease, SD standard deviation, XBCQ Xuan Bai Cheng Qi. 
Table 4 Total symptom scores in Xuan Bai Cheng Qi and control groups during 10 - day treatment

\begin{tabular}{|c|c|c|c|c|c|c|}
\hline \multirow[t]{2}{*}{ Total symptom scores } & \multicolumn{3}{|c|}{ Full analysis set } & \multicolumn{3}{|c|}{ Per - protocol analysis set } \\
\hline & $\begin{array}{c}X{ }^{a} Q^{a} \\
(n=122)\end{array}$ & $\begin{array}{l}\text { Control }^{a} \\
(n=122)\end{array}$ & $\begin{array}{l}\text { Treatment effect }^{\mathrm{b}} \\
\text { (95\% Cl; } P \text {-value) }\end{array}$ & $\begin{array}{c}X{ }^{a} Q^{a} \\
(n=122)\end{array}$ & $\begin{array}{l}\text { Control }^{a} \\
(n=122)\end{array}$ & $\begin{array}{l}\text { Treatment effect }^{\mathrm{b}} \\
\text { (95\% Cl; } P \text {-value) }\end{array}$ \\
\hline $1 \mathrm{~d}$ & $19.70(4.23)$ & $20.10(4.28)$ & $-0.39(-1.47,0.68 ; .471)$ & $19.64(4.24)$ & $20.09(4.32)$ & $-0.45(-1.56,0.67 ; .433)$ \\
\hline $3 d$ & $13.92(4,46)$ & $16.48(4.08)$ & $-2.57(-3.64,-1.49 ;<.001)$ & $13.82(4.50)$ & $16.42(3.95)$ & $-2.60(-3.70,-1.49 ;<.001)$ \\
\hline $5 d$ & $10.64(4.28)$ & $13.20(4.18)$ & $-2.56(-3.63,-1.49 ;<.001)$ & $10.59(4.24)$ & $13.12(4.10)$ & $-2.52(-3.61,-1.44 ;<.001)$ \\
\hline $7 d$ & $7.72(4.34)$ & $9.64(3.68)$ & $-1.92(-2.93,-0.90 ;<.001)$ & $7.64(4.38)$ & $9.54(3.65)$ & $-1.90(-2.95,-0.86 ;<.001$ \\
\hline $10 \mathrm{~d}$ & $5.21(4.43)$ & $7.00(3.72)$ & $-1.79(-2.82,-0.76 ; 0.001)$ & $5.03(4.29)$ & $6.92(3.74)$ & $-1.89(-2.93,-0.84 ;<.001)$ \\
\hline Mean $^{c}$ & $11.44(3.36)$ & $13.28(3.09)$ & $-1.84(-2.66,-1.03 ;<.001)$ & $11.34(3.40)$ & $13.22(3.05)$ & $-1.87(-2.71,-1.03 ;<.001)$ \\
\hline
\end{tabular}

Abbreviations: Cl confidence interval, XBCQ Xuan Bai Cheng Qi.

Mauchly's test of sphericity was applied: $\mathrm{W}=.506, P<.001$.

aData presented as mean (SD).

${ }^{\text {b }}$ Repeated-measures analysis of variance (ANOVA) for estimate with $95 \%$ confidence interval for difference

'Mean = change from baseline.

\section{Lung function}

$\mathrm{FEV}_{1}, \mathrm{FVC}$, and $\mathrm{FEV}_{1} \%$ pred were significantly higher over time in the Xuan Bai Cheng Qi group compared with the same parameters in the control group $\left(\mathrm{FEV}_{1}\right.$, FAS: mean difference $0.14,95 \%$ CI 0.06 to $0.22, P<.001$, PPS: mean difference $0.13,95 \%$ CI 0.06 to $0.21, P=.001$; FVC, FAS: mean difference $0.17,95 \%$ CI 0.06 to 0.28 , $P=.002$, PPS: mean difference $0.16,95 \%$ CI 0.05 to $0.27, P=.004 ; \mathrm{FEV}_{1} \%$ pred, FAS: mean difference 7.33 , 95\% CI 4.58 to $10.07, P<.001$, PPS: mean difference $7.20,95 \%$ CI 4.37 to $10.03, P<.001$ ) (Table 5).

\section{Arterial blood gas analysis}

At day $10, \mathrm{PaO}_{2}$ and $\mathrm{PaCO}_{2}$ were significantly improved in the Xuan Bai Cheng Qi group compared with $\mathrm{PaO}_{2}$ and $\mathrm{PaCO}_{2}$ in the control group $\left(\mathrm{PaO}_{2}\right.$, FAS: mean difference $4.75,95 \%$ CI 1.21 to $8.28, P=.009$, PPS: mean difference $4.08,95 \%$ IC 0.04 to $7.76, P=.030 ; \mathrm{PaCO}_{2}$, FAS: mean difference $-2.48,95 \%$ CI -4.53 to -0.44 , $P=.018$, PPS: mean difference $-2.69,95 \%$ CI -4.56 to $-0.81, P=.005)$. There was no significant difference between the Xuan Bai Cheng Qi and control groups in PH (FAS: mean difference $-0.00,95 \% \mathrm{CI}-0.01$ to 0.01 , $P=.894$, PPS: mean difference $-0.00,95 \%$ CI -0.01 to $0.01, P=.794$ ) (Table 6).

\section{Pro-inflammatory biomarkers (cytokines)}

Only 22 participants in the Xuan Bai Cheng Qi group and 16 participants in the control group permitted their blood to be collected at the end of the trial on day 10 . Therefore, comparison of biomarker levels between the two groups was based on these numbers of participants.

Table 5 Lung function measurements in Xuan Bai Cheng Qi and control groups before and after 10-day treatment

\begin{tabular}{|c|c|c|c|c|c|c|c|}
\hline \multirow{2}{*}{$\begin{array}{l}\text { Lung } \\
\text { function }^{\mathrm{a}}\end{array}$} & \multicolumn{3}{|c|}{$X_{B C Q^{b}}$} & \multicolumn{3}{|c|}{ Control $^{b}$} & \multirow{2}{*}{$\begin{array}{l}\text { Treatment effect }^{d} \\
(95 \% \mathrm{Cl} ; P \text { value) }\end{array}$} \\
\hline & $\begin{array}{c}\text { Day } 1 \\
\text { (Baseline) }\end{array}$ & $\begin{array}{l}\text { Day } 10 \\
\text { (End) }\end{array}$ & Mean $\mathrm{D}^{\mathrm{c}}$ & $\begin{array}{c}\text { Day } 1 \\
\text { (Baseline) }\end{array}$ & $\begin{array}{c}\text { Day } 10 \\
\text { (End) }\end{array}$ & Mean $D^{c}$ & \\
\hline \multicolumn{8}{|l|}{$\mathrm{FEV}_{1}$ (liters) } \\
\hline FAS $(122,122)$ & $1.18(0.41)$ & $1.44(0.49)$ & $0.24(0.29)$ & $1.19(0.40)$ & $1.29(0.47)$ & $0.10(0.31)$ & $0.14(0.06,0.22 ;<.001)$ \\
\hline PPS $(111,118)$ & $1.20(0.42)$ & $1.44(0.49)$ & $0.24(0.29)$ & $1.19(0.39)$ & $1.29(0.47)$ & $0.10(0.31)$ & $0.13(0.06,0.21 ; .001)$ \\
\hline \multicolumn{8}{|l|}{ FVC (liters) } \\
\hline FAS $(122,122)$ & $2.16(0.62)$ & $2.40(0.67)$ & $0.23(0.38)$ & $2.10(0.58)$ & $2.17(0.62)$ & $0.08(0.48)$ & $0.17(0.06,0.28 ; .002)$ \\
\hline PPS $(111,118)$ & $2.17(0.60)$ & $2.40(0.65)$ & $0.23(0.38)$ & $2.11(0.57)$ & $2.19(0.60)$ & $0.08(0.48)$ & $0.16(0.05,0.27 ; .004)$ \\
\hline \multicolumn{8}{|l|}{$\mathrm{FEV}_{1} \%$ pred } \\
\hline FAS $(122,122)$ & $51.54(19.49)$ & $62.39(19.57)$ & $10.59(11.76)$ & $52.13(19.17)$ & $55.35(20.59)$ & $3.22(10.53)$ & $7.33(4.58,10.07 ;<.001)$ \\
\hline PPS $(111,118)$ & $52.60(19.74)$ & $63.38(19.36)$ & 10.49 (11.84) & $52.17(19.27)$ & $55.56(22.68)$ & $3.39(10.50)$ & $7.20(4.37,10.03 ;<.001)$ \\
\hline
\end{tabular}

Abbreviations: $\mathrm{Cl}$ confidence interval, COPD chronic obstructive pulmonary disease, $F E V_{1}$ forced expiratory volume in one second, $F E V_{1} \%$ pred $\mathrm{FEV}_{1}$ percentage of predicted value, FVC forced vital capacity, GOLD Global Initiative for Chronic Obstructive Lung Disease, Mean D mean difference, XBCQ Xuan Bai Cheng Qi. ${ }^{\mathrm{a}} \mathrm{FAS}$ population was 122 in the XBCQ group and 122 in the control group; PPS population was 111 in the XBCQ group and 118 in the control group. ${ }^{\mathrm{b}}$ Data presented as mean (SD).

${ }^{c} P<.01$ : Change between pre-treatment and post-treatment (within group difference by paired t-test).

${ }^{\mathrm{d} C}$ Covariance analysis estimate with $95 \%$ confidence interval for difference. 
Table 6 Arterial blood gas analysis in Xuan Bai Cheng Qi and control groups before and after 10-day treatment

\begin{tabular}{|c|c|c|c|c|c|c|c|}
\hline \multirow[t]{2}{*}{ Variables $^{\mathrm{a}}$} & \multicolumn{3}{|c|}{$\mathrm{XBCQ}^{\mathrm{b}}$} & \multicolumn{3}{|c|}{ Control $^{b}$} & \multirow{2}{*}{$\begin{array}{l}\text { Treatment effect }^{d} \\
(95 \% \mathrm{Cl} ; P \text {-value) }\end{array}$} \\
\hline & $\begin{array}{c}\text { Day } 1 \\
\text { (Baseline) }\end{array}$ & $\begin{array}{l}\text { Day } 10 \\
\text { (End) }\end{array}$ & Mean D & $\begin{array}{c}\text { Day } 1 \\
\text { (Baseline) }\end{array}$ & Day 10 (End) & Mean D & \\
\hline \multicolumn{8}{|l|}{$\mathrm{PH}$} \\
\hline FAS $(122,122)$ & $7.41(0.05)$ & $7.41(0.04)$ & $0.01(0.05)$ & $7.42(0.05)$ & $7.42(0.05)$ & $-0.00(0.05)$ & $-0.00(-0.01,0.01 ; .894)$ \\
\hline PPS $(111,118)$ & $7.41(0.05)$ & $7.41(0.04)$ & $0.00(0.05)$ & $7.42(0.05)$ & $7.42(0.05)$ & $-0.00(0.05)$ & $-0.00(-0.01,0.01 ; .794)$ \\
\hline \multicolumn{8}{|l|}{$\mathrm{PaO}_{2}$} \\
\hline FAS $(122,122)$ & $75.56(19.38)$ & $85.69(16.42)$ & $13.13(18.12)^{c}$ & $71.47(18.90)$ & 80.49 (15.91) & $9.01(17.49)^{c}$ & $4.75(1.21,8.28 ; .009)$ \\
\hline PPS $(111,118)$ & $71.91(19.28)$ & $85.11(16.59)$ & $13.20(18.50)^{c}$ & $71.27(19.08)$ & $80.76(15.96)$ & $9.49(17.31)^{c}$ & $4.08(0.04,7.76 ; .030)$ \\
\hline \multicolumn{8}{|l|}{$\mathrm{PaCO}_{2}$} \\
\hline FAS $(122,122)$ & $47.41(10.04)$ & $39.52(11.55)$ & $-7.89(12.27)^{c}$ & $39.52(11.55)$ & $41.45(8.33)$ & $-4.76(8.97)^{\mathrm{c}}$ & $-2.48(-4.53,-0.44 ; .018)$ \\
\hline PPS $(111,118)$ & $46.46(13.32)$ & $38.93(9.47)$ & $-7.53(11.49)^{c}$ & $45.90(11.82)$ & $41.38(8.41)$ & $-4.52(8.83)^{c}$ & $-2.69(-4.56,-0.81 ; .005)$ \\
\hline
\end{tabular}

Abbreviations: $\mathrm{Cl}$ confidence interval, Mean $\mathrm{D}$ mean difference, $\mathrm{PaO}_{2}$ arterial partial pressure of oxygen, $\mathrm{PaCO}_{2}$ arterial partial carbon dioxide pressure, XBCQ Xuan Bai Cheng Qi.

${ }^{a}$ FAS population was 122 in the XBCQ group and 122 in the control group; PPS population was 111 in the XBCQ group and 118 in the control group.

${ }^{b}$ Data presented as mean (SD).

${ }^{C} P<.01$ : Change between pre - treatment and post - treatment (within group difference by paired $\mathrm{t}-$ test)

${ }^{\mathrm{d} C o v a r i a n c e}$ analysis estimate with $95 \%$ confidence interval for difference.

At day 10, TNF- $\alpha$, IL-4, IL-8, IL-1 $\beta$, IL-6, and IL-2 levels were significantly decreased in the Xuan Bai Cheng Qi group compared with levels in the control group (Table 7).

\section{Oxidant/antioxidant balance}

Based on serum collected from the 20 participants in the Xuan Bai Cheng Qi group and 20 participants in the control group, MDA level was significantly decreased in the Xuan Bai Cheng Qi group compared with that in the control group (mean difference -1.07 , 95\% CI -1.55 to $-0.59, P<.001$ ), while SOD level in the Xuan Bai Cheng Qi group was significantly higher than that in the control group (mean difference $6.09,95 \%$ CI 2.94 to 9.23, $P<.001)$ (Table 8).

\section{Safety variables}

Adverse events were recorded during the study period. Mild diarrhea occurred in six participants (4.92\%) in the Xuan Bai Cheng Qi group and in two participants (1.64\%) in the control group, but these incidents were not statistically significant $(P=.281)$. There were no significant differences in blood, urine, and liver and kidney function tests, or in electrocardiography results before and after the study period between the Xuan Bai Cheng Qi and control groups (Table 9).

\section{Adherence}

Adherence based on empty granule package count was high and similar in both the Xuan Bai Cheng Qi and control groups.

Table 7 Pro-inflammatory biomarker changes in Xuan Bai Cheng Qi and control groups before and after 10 - day treatment

\begin{tabular}{|c|c|c|c|c|c|c|c|}
\hline \multirow[t]{2}{*}{ Biomarkers } & \multicolumn{3}{|c|}{$X B C Q^{a}(n=22)$} & \multicolumn{3}{|c|}{ Control $^{a}(n=16)$} & \multirow{2}{*}{$\begin{array}{l}\text { Treatment effect }^{d} \\
(95 \% \mathrm{Cl} ; P \text {-value })\end{array}$} \\
\hline & $\begin{array}{c}\text { Day } 1 \\
\text { (Baseline) }\end{array}$ & $\begin{array}{l}\text { Day } 10 \\
\text { (End) }\end{array}$ & Mean $D^{c}$ & $\begin{array}{c}\text { Day } 1 \\
\text { (Baseline) }\end{array}$ & $\begin{array}{l}\text { Day } 10 \\
\text { (End) }\end{array}$ & Mean D & \\
\hline$\overline{T N F}-a(p g / m L)$ & $20.34(4.90)$ & $4.92(1.90)$ & $-15.42(4.88)$ & $18.90(3.61)$ & $6.47(1.56)$ & $-12.43(3.28)^{c}$ & $-1.70(-2.86,-0.54 ; .005)$ \\
\hline $\mathrm{IL}-2(\mathrm{pg} / \mathrm{mL})$ & $129.72(18.27)$ & $53.50(18.21)$ & $-76.23(25.21)$ & $129.54(28.70)$ & $84.10(11.01)$ & $-45.43(32.88)^{c}$ & $-30.60(-40.94,-20.25 ;<.001)$ \\
\hline $\mathrm{IL}-4(\mathrm{pg} / \mathrm{mL})$ & $5.98(1.49)$ & $2.36(0.56)$ & $-3.61(1.78)$ & $6.15(1.36)$ & $3.38(1.25)$ & $-2.77(1.70)^{c}$ & $-1.02(-1.65,-0.39 ; .002)$ \\
\hline $\mathrm{IL}-6(\mathrm{pg} / \mathrm{mL})$ & $3.95(0.80)$ & $2.06(0.53)$ & $-1.89(0.95)$ & $3.81(0.94)$ & $2.76(0.67)$ & $-1.06(0.48)^{c}$ & $-0.74(-1.20,-0.39 ;<.001)$ \\
\hline $\mathrm{IL}-8(\mathrm{pg} / \mathrm{mL})$ & $18.33(3.93)$ & $9.09(3.54)$ & $-9.24(6.11)$ & $18.01(4.17)$ & $14.92(3.86)$ & $-3.08(5.36)^{b}$ & $-5.80(-8.26,-3.34 ;<.001)$ \\
\hline $\mathrm{IL}-1 \beta(\mathrm{pg} / \mathrm{mL})$ & $13.42(2.12)$ & $8.36(1.02)$ & $-7.89(12.27)$ & $12.86(1.98)$ & $9.23(1.54)$ & $-4.76(8.97)^{c}$ & $-0.94(-1.78,-0.10 ; .029)$ \\
\hline
\end{tabular}

Abbreviations: $C l$ confidence interval, $I L-1 \beta$ interleukin -1 beta, $I L-2$ interleukin $-2, I L-4$ interleukin -4 , IL - 6 interleukin -6 , IL -8 interleukin - $8, M e a n ~ D$ mean difference, $T N F-a$ tumor necrosis factor alpha, XBCQ Xuan Bai Cheng Qi.

${ }^{\mathrm{a}}$ Data presented as mean (SD).

${ }^{\mathrm{b}} \mathrm{P}<.05$.

${ }^{c} P<.01$ : change between pre - treatment and post - treatment (within group difference by paired $t-$ test).

${ }^{d}$ Covariance analysis estimate with $95 \%$ confidence interval for difference. 
Table 8 Serum SOD and MDA in Xuan Bai Cheng Qi and control groups before and and after treatment

\begin{tabular}{|c|c|c|c|c|c|c|c|}
\hline \multirow[t]{2}{*}{ Variables } & \multicolumn{3}{|c|}{$X_{B C Q}^{a}(n=20)$} & \multicolumn{3}{|c|}{ Control $^{a}(n=20)$} & \multirow{2}{*}{$\begin{array}{l}\text { Treatment effect } \\
\text { (95\% Cl; } P \text { value })\end{array}$} \\
\hline & Day 1 (Baseline) & $\begin{array}{l}\text { Day } 10 \\
\text { (End) }\end{array}$ & Mean $D^{b}$ & $\begin{array}{c}\text { Day } 1 \\
\text { (Baseline) }\end{array}$ & $\begin{array}{l}\text { Day } 10 \\
\text { (End) }\end{array}$ & Mean D & \\
\hline SOD (U/mL) & $20.56(4.95)$ & $28.54(4.66)$ & $7.98(5.97)$ & $21.90(5.32)$ & $23.24(6.76)$ & $1.33(4.21)$ & $6.09(2.94,9.23 ;<.001)$ \\
\hline MDA (nmol/mL) & $4.43(1.52)$ & $1.89(0.52)$ & $-2.54(1.65)$ & $4.52(1.44)$ & $2.98(1.45)$ & $-1.54(0.94)^{b}$ & $-1.07(-1.55,-0.59 ;<.001)$ \\
\hline
\end{tabular}

Abbreviations: $C l$ confidence interval, MDA malondialdehyde, Mean D mean difference, SOD superoxide dismutase, XBCQ Xuan Bai Cheng Qi

${ }^{a}$ Data presented as mean (SD).

${ }^{\mathrm{b}} P<.01$ : Change between pre-treatment and post-treatment (within group difference by paired t-test).

${ }^{c}$ Covariance analysis estimate with $95 \%$ confidence interval for difference.

\section{Discussion}

This multicenter, randomized, double-blind, placebocontrolled study showed that as an adjuvant treatment Xuan Bai Cheng Qi formula produced a significant improvement in symptoms associated with AECOPD of the TCM syndrome type, phlegm-heat obstructing the lungs. Total symptom scores from baseline showed that Xuan Bai Cheng Qi ameliorated cough, phlegm, wheezing, and chest congestion. Lung function and blood gas levels were also significantly improved in the intervention group compared with the control group.

AECOPD appears to be triggered by increased airway inflammation. A number of pro-inflammatory cytokines, such as TNF- $\alpha$, IL-4, IL-8, IL-1 $\beta$, IL-6, IL-2, have been detected during COPD exacerbation and have been reported to be increased in both sputum and serum during COPD exacerbations [21-25]. In our study, levels of pro-inflammatory mediators in the Xuan Bai Cheng Qi group were lower than those in the control group. We propose that as an adjuvant treatment, Xuan Bai Cheng Qi contributed to regulating systemic inflammatory response. Previous studies have shown that the active ingredients in the herbs that comprise Xuan Bai Cheng Qi formula, such as emodin in Rheum officinale Baill [26], calcium sulfate in Gypsum fibrosum [27], triterpenoid saponins in Trichosanthes kirilowii Maxim [28], and amygdalin in Semen Armeniacae amarum [29] possess anti-inflammatory effects.

Oxidative stress is another component in the pathogenesis of AECOPD [30-32]. Oxidative stress can induce extensive tissue damage and activate the nuclear factor kappa-light-chain-enhancer of activated B cells $(\mathrm{NF}-\mathrm{kB})$ pathway, which provokes the release of proinflammatory molecules, such as TNF- $\alpha$, IL-8. Our results indicate that compared with the control group, SOD activity was higher and MDA content was lower in the Xuan Bai Cheng Qi group. Thus Xuan Bai Cheng Qi may contribute to restoring oxidant/antioxidant balance.

Mention needs to be made that of the 122 participants each in the Xuan Bai Chang Qi and control groups, only 20 and 16 participants, respectively, allowed their serum to be collected at the end of the trial for evaluation of biomarker changes. A similar situation occurred during

Table 9 Safety profile

\begin{tabular}{|c|c|c|c|c|c|}
\hline \multirow[t]{2}{*}{ Variables $^{\mathrm{a}}$} & \multicolumn{2}{|c|}{$\mathrm{XBCQ}^{\mathrm{b}}(\mathrm{n}=122)$} & \multicolumn{2}{|c|}{ Control $^{b}(n=122)$} & \multirow[t]{2}{*}{$P$-value } \\
\hline & $\begin{array}{c}\text { Day } 1 \\
\text { (Baseline) }\end{array}$ & $\begin{array}{c}\text { Day } 10 \\
\text { (End) }\end{array}$ & $\begin{array}{c}\text { Day } 1 \\
\text { (Baseline) }\end{array}$ & $\begin{array}{c}\text { Day } 10 \\
\text { (End) }\end{array}$ & \\
\hline WBC $\left(10^{9} / \mathrm{L}\right)$ & $8.33(3.49)$ & $7.51(2.81)$ & $9.38(7.95)$ & $7.53(3.11)$ & .843 \\
\hline $\mathrm{HB}(\mathrm{g} / \mathrm{L})$ & $138.2(24.0)$ & $138.6(31.0)$ & $136.3(28.1)$ & $134.3(28.1)$ & .310 \\
\hline $\operatorname{RBC}\left(10^{12} / \mathrm{L}\right)$ & $4.39(0.65)$ & $4.38(0.64)$ & $4.33(0.61)$ & $4.27(0.57)$ & .263 \\
\hline $\operatorname{PLT}\left(10^{9} / \mathrm{L}\right)$ & $198.4(62.4)$ & $210.4(78.7)$ & $209.4(76.9)$ & $207.5(68.4)$ & .178 \\
\hline NEUT (\%) & $69.6(14.4)$ & $64.7(12.9)$ & $68.9(14.9)$ & $62.1(13.4)$ & .111 \\
\hline ALT (U/L) & $21.0(11.1)$ & $22.2(12.1)$ & $21.9(16.3)$ & $22.5(14.1)$ & .920 \\
\hline $\mathrm{BUN}(\mathrm{mmol} / \mathrm{L})$ & $5.6(1.6)$ & $5.7(2.4)$ & $6.1(2.8)$ & $5.8(2.8)$ & .511 \\
\hline $\mathrm{CR}(\mu \mathrm{mmol} / \mathrm{L})$ & $75.4(20.4)$ & $74.0(21.5)$ & $85.2(46.5)$ & $80.3(35.9)$ & .863 \\
\hline $\operatorname{BUN}(n)^{c}$ & $27(122)$ & $32(122)$ & $15(122)$ & $7(122)$ & .116 \\
\hline ECG $(n)^{c}$ & $56(122)$ & $45(122)$ & $57(122)$ & $47(122)$ & .895 \\
\hline
\end{tabular}

Abbreviations: $A L T$ alanine aminotransferase, BUN blood urea nitrogen, $C r$ creatinine, ECG electrocardiogram, $H B$ hemoglobin, NEUT neutrophilic granulocyte, PLT platelets, $R B C$ red blood cell, WBC white blood cell.

${ }^{a}$ Covariance analysis for between group after treatment.

${ }^{b}$ Data presented as mean (SD).

${ }^{\mathrm{C}} \mathrm{Chi}$-square test for between group after treatment. 
assessment of levels of SOD and MDA, with only 20 participants in each group permitting serum collection. For ethical considerations, we respected participants' inclination. Nevertheless, based on the results we did obtain, there were significant differences between the two groups as described above.

Xuan Bai Cheng Qi formula appears to be safe. Serious untoward effects were not reported during the study. Six participants in the Xuan Bai Cheng Qi group experienced mild diarrhea. Toxicological studies on the active ingredient in individual medicinals in Xuan Bai Cheng Qi formula have shown that long-term use of Rheum officinale Baill may induce liver degeneration [33]; toxicity of Semen Armeniacae amarum is mainly from amygdaloside, which can produce the toxic hydrocyanic acid by enzymatic hydrolysis [34]; Trichosanthes kirilowii Maxim is not known to be toxic [35]; and Gypsum fibrosum is mildly toxic [36]. Typically, during processing of raw medicinals and decoction of the combined medicinals, it is likely that the individual toxic effects are reduced or even neutralized [37].

The main limitation of this study is its short duration of 10 days. Changes in frequency of acute exacerbations of COPD were not observable. In addition, the comparison between biomarkers and levels of SOD and MDA are of an observational nature, which may therefore be biased. Further studies should be performed to evaluate the long-term efficacy of Xuan Bai Cheng Qi formula as an adjuvant treatment for AECOPD.

\section{Conclusions}

Traditional Chinese medicinals are commonly used to treat lung conditions, including acute exacerbation of chronic obstructive pulmonary disease (AECOPD). However, there is a lack of modern research to validate such empirical remedies. To the best of our knowledge, this study is the first large-scale, multicenter randomized control trial evaluating the efficacy and safety in humans of the traditional formula Xuan Bai Cheng Qi as an adjuvant treatment for AECOPD of the TCM syndrome type, phlegm-heat blocking the lungs. Our results found that compared with placebo, Xuan Bai Cheng Qi appears to be safe and effective for AECOPD with improvement in symptoms as well as biomarker parameters.

\section{Abbreviations \\ COPD: Chronic obstructive pulmonary disease; TCM: Traditional Chinese medicine; AECOPD: Acute exacerbation of chronic obstructive pulmonary disease; FVC: Forced vital capacity; FEV 1 : Forced expiratory volume in one second; FEV $\%$ pred: FEV 1 percentage of predicted value; SOD: Superoxide dismutase; MDA: Malondialdehyde.}

\section{Competing interests}

The authors declare that they have no competing interests.

\section{Authors' contributions}

$Y L$ and $X Z$ conceived and designed the study. RW, YS, and JZ input the data, and $M L$ and $F Z$ analysed the data and drafted the manuscript. All authors read and approved the final manuscript.

\section{Acknowledgments}

This study was funded by China National key basic research plan (No. 2009(B522704).

The authors particularly thank Professor Jianping Liu and the Centre for Evidence-Based Chinese Medicine, Beijing University of Chinese Medicine, for their valuable contribution to this study.

We appreciate the assistance and support in patient recruitment from the following individuals: Limei Geng (Hebei Provincial Hospital of Chinese Medicine), Hong Li (University Hospital of Gansu Traditional Chinese Medicine), Huiping Su (Dongzhimen Hospital affiliated to Beijing University of Chinese Medicine), Jinmin Liu (Dongfang Hospital affiliated to Beijing University of Chinese Medicine), Qixiang Wu (Henan Province Kaifeng City Hospital of Traditional Chinese Medicine) Xudong Xiong (Shuguang Hospital affiliated to Shanghai University of Chinese Medicine), Yanling Xu (Affiliated Hospital of Liaoning University of Traditional Chinese Medicine), Nianzhi Zhang (First Affiliated Hospital of Anhui University of Chinese Medicine), and all the medical staff who participated in this study.

We are indebted to Nissi S. Wang, MSc, for reviewing and editing the manuscript.

Received: 27 July 2013 Accepted: 1 July 2014

Published: 11 July 2014

\section{References}

1. Mathers CD, Loncar D: Projections of global mortality and burden of disease from 2002 to 2030. PLoS Med 2006, 3(11):e442.

2. Bourbeau J, Ford G, Zackon H, Pinsky N, Lee J, Ruberto G: Impact on patients' health status following early identification of a COPD exacerbation. Eur Respir J 2007, 30:907-913.

3. Donaldson GC, Seemungal TA, Bhowmik A, Wedzicha JA: Relationship between exacerbation frequency and lung function decline in chronic obstructive pulmonary disease. Thorax 2002, 57:847-852.

4. Donaldson GC, Wedzicha JA: COPD exacerbations.1: Epidemiology. Thorax 2006, 61:164-168.

5. Global Initiative for Chronic Obstructive Lung Disease, Inc: Global strategy for the diagnosis, management, and prevention of chronic obstructive pulmonary disease (updated 2013). Available at: http://www.goldcopd.org/ uploads/users/files/GOLD_Report_2013_Feb20.pdf. Accessed January 27, 2014

6. Xu WJ, Wang TF, Wang ZY, Jiao Y, Su HP, Yu LL, Lu JC, Zou XJ, Zhang N, Chen XH, Miao JY, Xu YL, Li SY, Wang MH, Wang QG: Comparison of traditional Chinese symptomatologies of acute and stable stages of chronic obstructive pulmonary disease. Acta Universitatis Traditionis Medicalis Sinensis Pharmacologiaeque Shanghai 2010, 24(6):35-39. Chinese.

7. Xin DY: Disseminating lung qi unblocking the bowels method in the treatment of acute exacerbation of chronic obstructive pulmonary disease: a randomized controlled observation. J Pract Tradit Chinese Intern Med 2012, 26(12):25-28. Chinese.

8. Li YH, Zhong XG, Jia X, Sun Y, Zheng FJ, Wang W, Zhou XW, Zhu XH, Tian Y, Liu XH: Effects of "catharsis large intestine"on oxidative stress in rats with COPD. Chin J Tradit Chin Med Pharm 2010, 25:1196-1198. Chinese.

9. Wang $\mathrm{T}$, Zhang $\mathrm{Q}$, Li YH, Xie H, Zhong XG, Jia X, Liu M, Liu XH: J Beijing Univ Tradit Chin Med 2013, 36:104-107. Chinese.

10. Meng FS: Clinical study on Xuan Bai Cheng Qi formula for acute exacerbation of chronic obstructive pulmonary disease. J New Chin Med 2012, 44:23-24. Chinese.

11. Shi YJ, Wang H: Clinical observation of Tongfu treatment on treating 40 COPD patients with respiratory insufficiency. Med Innov China 2013, 17:39-40. Chinese

12. Global Initiative for Chronic Obstructive Lung Disease, Inc: Global strategy for the diagnosis, management, and prevention of chronic obstructive lung disease (updated 2007). Available at: http://www.goldcopd.org/ uploads/users/files/GOLDReport07_0108.pdf. Accessed February 7, 2014.

13. Chinese Society of Respiratory Diseases: Chronic obstructive pulmonary disease diagnostic and treatment guidelines (revised 2007). Chin J Tuberc Respir Dis 2007, 30:8-17. Chinese. 
14. State Administration of Traditional Chinese Medicine: Standards of Traditional Chinese Syndrome Diagnosis and Treatment. Beijing, China: China Press of Traditional Chinese Medicine; 1994:2.

15. Wang YY, Lu ZL: Pulmonary disease. In Internal Medicine of Traditional Chinese Medicine. 2nd edition. Beijing: People's Medical Publishing House; 2002:200. Chinese.

16. Jamieson S: Likert scales: how to (ab)use them. Med Educ 2004, 38:1217-1218

17. Zheng XY, Zheng XY: Respiratory disease. In Guide to New Traditional Chinese Medicine Research (Trial), Volume 1. Edited by Zheng XY. Beijing: China Medical Science Press; 2002:53-58. Chinese.

18. Li W, Mao B, Wang G, Wang L, Chang J, Zhang Y, Wan MH, Guo J: A study of the mechanism of Qingre Huatan therapy in treatment of acute exacerbation of chronic obstructive pulmonary disease by improving airway inflammation and mucus hypersecretion. J Chin Integr Med 2008, 6:799-805. Chinese.

19. Peng WB, Li SF: Clinical observation of lung-purging, blood-activating and fu-unblocking herbs with western medicine in treating acute exacerbation of chronic obstructive pulmonary disease. Shanghai J Tradit Chin Med 2009, 07:28-30. Chinese.

20. Chen $\mathrm{H}$, Zhi KY, Feng WJ: Clinical observation of fu-unblocking, clearing-heat in treating 128 patients with acute exacerbation of chronic obstructive pulmonary disease. Shanxi J Tradit Chin Med 2008, 06:29-30. Chinese.

21. Papi A, Luppi F, Franco F, Fabbri LM: Pathophysiology of exacerbations of chronic obstructive pulmonary disease. Proc Am Thorac Soc 2006, 3:245-251.

22. Mallia P, Johnston SL: Mechanisms and experimental models of chronic obstructive pulmonary disease exacerbations. Proc Am Thorac Soc 2005, 2:361-366.

23. Ko FWS, Leung TF, Wong GWK, Ngai J, To KW, Ng S, Hui DSC: Measurement of tumor necrosis factor-alpha, leukotriene B4, and interleukin 8 in the exhaled breath condensate in patients with acute exacerbations of chronic obstructive pulmonary disease. Int J Chron Obstruct Pulmon Dis 2009, 4:79-86.

24. Almansa R, Sanchez-Garcia M, Herrero A, Calzada S, Roig V, Barbado J, Rico L, Bobillo F, Eiros JM, Iglesias V, de Lejarazu RO, Bermejo-Martin JF: Host response cytokine signatures in viral and nonviral acute exacerbations of chronic obstructive pulmonary disease. J Interferon Cytokine Res 2011, 31:409-413.

25. Liang R, Zhang W, Song YM: Levels of leptin and IL-6 in lungs and blood are associated with the severity of chronic obstructive pulmonary disease in patients and rat models. Mol Med Rep 2013, 7:1470-1476.

26. Chu X, Wei M, Yang X, Cao Q, Xie X, Guan M, Wang D, Deng X: Effects of an anthraquinone derivative from Rheum officinale Baill, emodin, on airway responses in a murine model of asthma. Food Chem Toxicol 2012, 50:2368-2375.

27. Zhou YX, Li M, Tang ZS, Wang B, Zhang B: Gypsum compounds and their antipyretic function and anti-inflammatory mechanisms. J Shaanxi Coll of Tradit Chin Med 2012, 35(5):74-76.

28. Bhattacharya S, Haldar PK: Protective role of the triterpenoid-enriched extract of Trichosanthes dioica root against experimentally induced pain and inflammation in rodents. Nat Prod Res 2012, 26:2348-2352.

29. Yang HY, Chang HK, Lee JW, Kim YS, Kim H, Lee MH, Shin MS, Ham DH, Park HK, Lee H, Kim CJ: Amygdalin suppresses lipopolysaccharide-induced expressions of cyclooxygenase-2 and inducible nitric oxide synthase in mouse BV2 microglial cell. Neurol Res 2007, 29(11):59-64

30. Skyba P, Kluchova Z, Joppa P, Petrasova D, Tkacova R: Nutritional status in relation to respiratory impairment and systemic inflammation in patients with acute exacerbations of COPD. Med Sci Monit 2009, 15(10):CR528-CR533.

31. Zeng M, Li Y, Jiang Y, Lu G, Huang X, Guan K: Local and systemic oxidative stress status in chronic obstructive pulmonary disease patients. Can Respir J 2013, 20(1):35-41.

32. Stanojkovic I, Kotur-Stevuljevic J, Milenkovic B, Spasic S, Vujic T, Stefanovic A, Llic A, Ivanisevic J: Pulmonary function, oxidative stress and inflammatory markers in severe COPD exacerbation. Respir Med 2011, 105(Suppl 1):S31-S37.

33. Yu CL, Huang TK, Ding ZZ, Gao XL, Zhang ZD: Traditional Chinese Medicine Thesauruses, Volume 1. 1st edition. Beijing: China Medical Science Press: 1993:327.
34. Yu CL, Huang TK, Ding ZZ, Gao XL, Zhang ZD: Traditional Chinese Medicine Thesauruses, Volume 2. 1st edition. Beijing: China Medical Science Press; 1993:159.

35. Yu CL, Huang TK, Ding ZZ, Gao XL, Zhang ZD: Traditional Chinese Medicine Thesauruses, Volume 2. 1st edition. Beijing: China Medical Science Press; 1993:1985.

36. Yu CL, Huang TK, Ding ZZ, Gao XL, Zhang ZD: Traditional Chinese Medicine Thesauruses, Volume 1. 1st edition. Beijing: China Medical Science Press; 1993:1482.

37. Chan K: Chinese herbal medicine concepts of herbs. In Interactions Between Chinese Herbal Medicinal Products and Orthodox Drugs. Edited by Chan K, Cheung L. London: Taylor and Francis; 2000:36-41.

\section{doi:10.1186/1472-6882-14-239}

Cite this article as: Liu et al: Xuan Bai Cheng Qi formula as an adjuvant treatment of acute exacerbation of chronic obstructive pulmonary disease of the syndrome type phlegm-heat obstructing the lungs: a multicenter, randomized, double-blind, placebo-controlled clinical trial. BMC Complementary and Alternative Medicine 2014 14:239.

\section{Submit your next manuscript to BioMed Central and take full advantage of:}

- Convenient online submission

- Thorough peer review

- No space constraints or color figure charges

- Immediate publication on acceptance

- Inclusion in PubMed, CAS, Scopus and Google Scholar

- Research which is freely available for redistribution

Submit your manuscript at www.biomedcentral.com/submit
C Biomed Central 